\title{
The structure and dynamics of a large-scale plasmoid generated by fast reconnection in the geomagnetic tail
}

\author{
M. Ugai \\ Research Center for Space and Cosmic Evolution, Ehime University, Matsuyama 790-8577, Japan \\ Received: 24 November 2010 - Revised: 27 December 2010 - Accepted: 4 January 2011 - Published: 13 January 2011
}

\begin{abstract}
As a sequence of Ugai (2010b), the present paper studies in detail the structure and dynamics of large-scale (principal) plasmoid, generated by the fast reconnection evolution in a sheared current sheet with no initial northward field component. The overall plasmoid domain is divided into the plasmoid reconnection region $\mathrm{P}$ and the plasmoid core region $\mathrm{C}$. In the region $\mathrm{P}$, the magnetized plasma with reconnected field lines are accumulated, whereas in the region $\mathrm{C}$, the plasma, which was intially embedded in the current sheet and has been ejected away by the reconnection jet, is compressed and accumulated. In the presence of the sheared magnetic field in the east-west direction in the current sheet, the upper and lower parts of the reconnection region $\mathrm{P}$ are inversely shifted in the east-west directions. Accordingly, the plasmoid core region $\mathrm{C}$ with the accumulated sheared field lines is bent in the north-south direction just ahead of the plasmoid center $x=X_{\mathrm{C}}$, causing the magnetic field component in the north-south direction, whose sign is always opposite to that of the reconnected field lines. Therefore, independently of the sign of the initial sheared field, the magnetic field component $B_{\mathrm{Z}}$ in the north-south direction has the definite bipolar profile around $X_{\mathrm{C}}$ along the $\mathrm{x}$-axis. At $x=X_{\mathrm{C}}$, the sheared field component has the peak value, and as the sheared fields accumulated in the region $\mathrm{C}$ become larger, the bipolar field profile becomes more distinct.
\end{abstract}

Keywords. Magnetospheric physics (Magnetotail; Storms and substorms)

\section{Introduction}

Magnetic reconnection is essential for large dissipative events in space plasmas, such as solar flares and geomagnetic substorms (Nishida, 1989; Priest and Forbes, 2000; Runov et

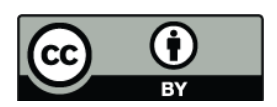

Correspondence to: M. Ugai

(ugai@cosmos.ehime-u.ac.jp) al., 2003; Klimchuk, 2006; Nakamura et al., 2006; Sharma et al., 2008). In view of the large-scale explosive magnetic energy conversion, we have proposed the spontaneous fast reconnection model. The details of this model have already been reported in our previous papers (e.g., Ugai, 2008, and references therein). It is demonstrated that the condition for the fast reconnection evolution is that the current sheet width in the sheet current direction is about three times larger than its thickness. In the geomagnetic tail, the current sheet is formed by the interaction between the Earth's dipole field and the solar wind. Once such a thin current sheet is formed, initiated by a disturbance, extreme current sheet thinning (current concentration) occurs around an $\mathrm{X}$ neutral line in collisionless plasmas, so that current-driven anomalous resistivities should necessarily be caused by plasma microturbulences such as ion-acoustic instabilities (Lui, 2001, 2004; Petkaki and Freeman, 2008). In fact, we find that the fast reconnection evolution is little influenced by functional forms nor parameter values of current-driven anomalous resistivity model. In other words, the fast reconnection mechanism can readily build up spontaneously in such thin current sheets once they are formed in space (collisionless) plasmas.

Regarding the substorm triggering mechanism, an essential question is to clarify how the fast reconnection mechanism can build up in the geomagnetic tail. Figure 1 schematically illustrates the cross section of the tail current sheet with the coordinate axes employed in the present study, where the $\mathrm{x}-, \mathrm{y}-$ and $\mathrm{z}$-axes are directed to the Earthward, duskward and northward directions, respectively. Here, the magnetic fields are assumed to be antiparallel in the x-direction. The tail current sheet configuration may be controlled by solar wind conditions, and then the spontaneous fast reconnection model predicts that once the current sheet becomes so thin that the effective current sheet width $2 \mathrm{~W}$ in the sheet current direction becomes about three times larger than the effective current sheet thickness $2 d_{0}, W>3 d_{0}$ say, the fast reconnection mechanism can drastically be triggered and evolve spontaneously. In a sheared current sheet, the fast reconnection

Published by Copernicus Publications on behalf of the European Geosciences Union. 


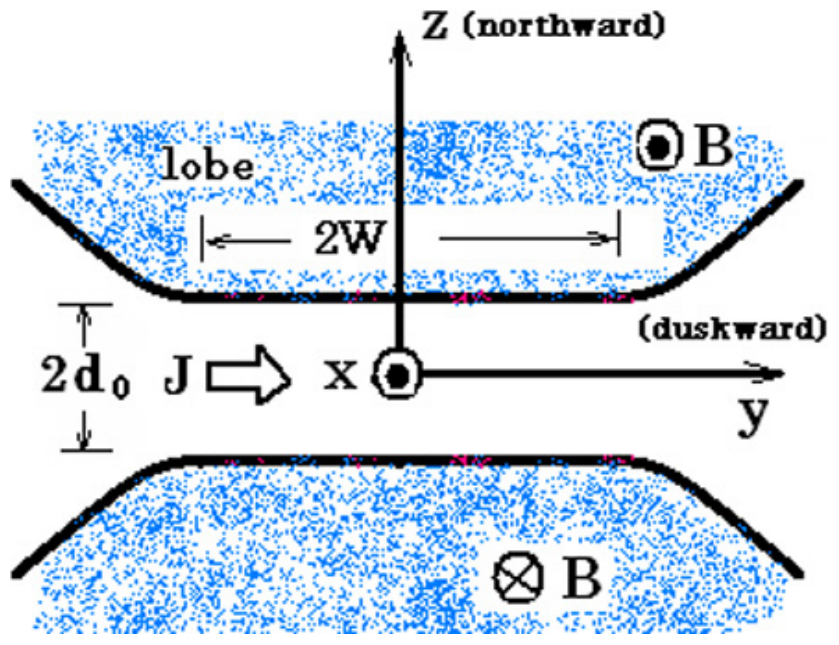

Fig. 1. Schematic drawing of the tail current sheet in the (y,z)-plane, where the $\mathrm{x}-, \mathrm{y}$ - and $\mathrm{z}$-axes, employed in the present study, are directed to the Earthward, duskward and northward directions, respectively, consistent with the conventional GSM coordinates; also, $\boldsymbol{J}$ is the sheet current, and $2 W$ and $2 d_{0}$ indicate the effective current sheet width and thickness, respectively.

evolution becomes harder for the larger sheared field, and in a force-free current sheet the condition for the fast reconnection evolution is that the current sheet width is about six times larger than its thickness, $W>6 d_{0}$ say (Ugai, 2010a).

One of the most fundamental questions on substorms may be to clarify the physical mechanism of plasmoid traveling in the tail. The traditional plasmoid picture predicts that if the reconnection starts somewhere in the tail current sheet, where a northward magnetic field usually exists, a plamoid structure with closed or spiral field lines may be formed in the tailward direction (Hones Jr., 1977), which was examined by an MHD simulation (Birn and Hones Jr., 1981). However, in this picture, the $\mathrm{x}$-directional plasmoid dimension may simply be determined by that of the outermost closed (spiral) field lines without considering the essential reconnection dynamics. On the contrary, the two-dimensional (2-D) plasmoid structure was studied on the basis of the spontaneous fast reconnection model (Ugai, 1995; Abe and Hoshino, 2001). Recently, we demonstrate that even without any initial northward magnetic field the magnetic field profiles inside the plasmoid, generated by the fast reconnection mechanism in the sheared current sheet, are, both qualitatively and quantitatively, in good agreement with the satellite observations; in particular, the north-south field component has the definite bipolar structure around the plasmoid center, where the east-west field component has the peak value (Ugai, 2010b). Also, the similar results are reported with a different numerical scheme by Shirataka et al. (2006) on the basis of the spontaneous fast reconnection model, and similar structures are reported in Cluster observations (Runov et al., 2008). Hence, the main theme of the present paper is to ex- amine the structure and dynamics of the large-scale (principal) plasmoid in more detail and to understand why and how the basic magnetic field profiles are generated inside the plasmoid. Here, the "plasmoid" means the plasma sheet bulge that is directly generated by the fast reconnection jet, which is quite different from the so-called "flux rope" generated by multiple reconnections. We also distinguish the "large-scale (principal) plasmoid" from the small-scale magnetic islands that are usually generated in the active $\mathrm{X}$ reconnection (diffusion) region and propagate in the central plasma sheet (Ugai and Zheng, 2006). In the present study, no northward magnetic field is considered in the initial current sheet.

\section{Simulation modeling}

The present simulation model is quite the same as Ugai (2010b), except that the results are shown with the coordinate system in Fig. 1. The details of the numerical procedure are already described in Ugai (2008), and all the present simulation results can readily be confirmed.

\subsection{Basic equations}

The compressible magnetohydrodynamic (MHD) equations are

$D \rho / D t=-\rho \nabla \cdot \boldsymbol{u}, \quad \rho D \boldsymbol{u} / D t=-\nabla P+\boldsymbol{J} \times \boldsymbol{B}$,

$\partial \boldsymbol{B} / \partial t-\nabla \times(\boldsymbol{u} \times \boldsymbol{B})=-\nabla \times(\eta \boldsymbol{J})$,

$\rho D e / D t=-P \nabla \cdot \boldsymbol{u}+\eta \boldsymbol{J}^{2}$,

$\boldsymbol{J}=\nabla \times \boldsymbol{B} / \mu_{0}, \quad \nabla \cdot \boldsymbol{B}=0$,

where $D / D t \equiv \partial / \partial t+\boldsymbol{u} \cdot \nabla$; the gas law, $P=(\gamma-1) \rho e$, is assumed ( $e$ is the internal energy per unit mass, and $\gamma$ is the specific heat ratio with $\gamma=5 / 3$ assumed here (an adiabatic case)), as is Ohm's law, $\boldsymbol{E}+\boldsymbol{u} \times \boldsymbol{B}=\eta \boldsymbol{J}$ ( $\eta$ may be an effective resistivity). The basic Eqs. (1) are transformed to a conservation-law form, and the modified Lax-Wendroff scheme is used for the numerical computation (Ugai, 2008).

\subsection{Initial-boundary conditions}

Initially, a long current sheet with sheared antiparallel magnetic fields $\left(B_{\mathrm{x}}\right)$ is assumed. Then, the normalization of quantities, based on the initial quantities, is self-evident: distances are normalized by the z-directional current sheet halfthickness $d_{0}$, magnetic field $\boldsymbol{B}$ by $B_{\mathrm{x} 0}$, plasma pressure $P$ by $P_{0}=B_{\mathrm{x} 0}^{2} /\left(2 \mu_{0}\right)$; also, plasma density $\rho$ by $\rho_{i}=\rho(z=0)$, plasma flow velocity $\boldsymbol{u}$ by $V_{A x 0}\left(=B_{\mathrm{x} 0} / \sqrt{\mu_{0} \rho_{i}}\right)$, time $t$ by $d_{0} / V_{A x 0}$. The Alfvén velocity based on $B_{\mathrm{x} 0}$ in the ambient magnetic field region is given by $V_{\mathrm{Ae}}=V_{A x 0} / \sqrt{\rho_{e}}\left(\rho_{e}\right.$ is the plasma density in the magnetic field region).

The magnetic field $\boldsymbol{B}=\left[B_{\mathrm{x}}(z), B_{\mathrm{y}}(z), 0\right]$ is initially assumed as: $B_{\mathrm{x}}(z)=\sin (\pi z / 2)$ for $0<z<1 ; B_{\mathrm{x}}=1$ for 
$1<z<5 ; B_{\mathrm{x}}=\cos [(z-5) \pi / 0.6]$ for $5<z<5.3 ; B_{\mathrm{X}}=0$ for $z>5.3$; also, $B_{\mathrm{x}}(z)=-B_{\mathrm{x}}(-z)$ for $z<0$. The sheared field component $B_{\mathrm{y}}$ is initially assumed as: $B_{\mathrm{y}}(z)=B_{\mathrm{y} 0}+$ $\alpha \cos (z \pi / 2)$ for $|z|<1$ and $B_{\mathrm{y}}=B_{\mathrm{y} 0}$ for $|z|>1$. Here, $B_{\mathrm{y} 0}$ and $\alpha$ are taken to be parameters for the initial sheared field. Fluid velocity $\boldsymbol{u}=(0,0,0)$, and plasma pressure $P(z)$ satisfies the pressure-balance condition,

$P+B_{\mathrm{x}}^{2}+B_{\mathrm{y}}^{2}=1+\beta_{0}+B_{\mathrm{y} 0}^{2}$,

where $\beta_{0}$ is the ratio of the plasma pressure to the magnetic pressure $B_{\mathrm{x} 0}^{2} /\left(2 \mu_{0}\right)$ in the magnetic field region $1<z<5$ (here, $\beta_{0}=0.15$ is taken). Also, plasma density $\rho$ initially satisfies

$\rho(z)=P(z) /\left(1+\beta_{0}+B_{\mathrm{y} 0}^{2}-\left(B_{\mathrm{y} 0}+\alpha\right)^{2}\right)$,

so that $\rho_{e}=\beta_{0} /\left(1+\beta_{0}+B_{\mathrm{y} 0}^{2}-\left(B_{\mathrm{y} 0}+\alpha\right)^{2}\right)$ in the ambient magnetic field region, and the initial temperature $T=P / \rho$ is, for simplicity, assumed to be constant everywhere.

The computational region is taken to be a rectangular box, $0 \leq x \leq L_{\mathrm{x}}, 0 \leq z \leq L_{\mathrm{z}}$, and $-L_{\mathrm{y}} \leq y \leq L_{\mathrm{y}}$, and the axis symmetry boundary conditions are assumed as follows (Ugai, 2010a, b). With respect to the x-axis, $\rho(x,-y,-z)=\rho(x, y, z), \quad u_{\mathrm{x}}(x,-y,-z)=u_{\mathrm{x}}(x, y, z)$, $u_{\mathrm{y}}(x,-y,-z)=-u_{\mathrm{y}}(x, y, z), u_{\mathrm{z}}(x,-y,-z)=-u_{\mathrm{z}}(x, y, z)$, $B_{\mathrm{x}}(x,-y,-z)=-B_{\mathrm{x}}(x, y, z), B_{\mathrm{y}}(x,-y,-z)=B_{\mathrm{y}}(x, y, z)$, $B_{\mathrm{Z}}(x,-y,-z)=B_{\mathrm{Z}}(x, y, z)$, and $P(x,-y,-z)=P(x, y, z)$, for a fixed value of $x$ in $0 \leq x \leq L_{\mathrm{x}}$. With respect to the z-axis, $\rho(-x,-y, z)=\rho(x, y, z)$, $u_{\mathrm{x}}(-x,-y, z)=-u_{\mathrm{x}}(x, y, z), u_{\mathrm{y}}(-x,-y, z)=-u_{\mathrm{y}}(x, y, z)$, $u_{\mathrm{z}}(-x,-y, z)=u_{\mathrm{z}}(x, y, z), \quad B_{\mathrm{X}}(-x,-y, z)=B_{\mathrm{X}}(x, y, z)$, $B_{\mathrm{y}}(-x,-y, z)=B_{\mathrm{y}}(x, y, z), B_{\mathrm{z}}(-x,-y, z)=-B_{\mathrm{z}}(x, y, z)$, and $P(-x,-y, z)=P(x, y, z)$, for a fixed value of $z$ in $0 \leq z \leq L_{\mathrm{z}}$. On the other boundary planes $\left(x=L_{\mathrm{x}}, y=L_{\mathrm{y}}, y=-L_{\mathrm{y}}, z=L_{\mathrm{z}}\right)$, the free boundary conditions are assumed, so that the reconnection ouflow is open to the free space.

The effective resistivity for the Ohm's law (normalized by $\left.\mu_{0} d_{0} V_{A x 0}\right)$ is assumed to be,

$$
\begin{aligned}
\eta(\boldsymbol{r}, t) & =k_{\mathrm{d}}\left[V_{\mathrm{d}}(\boldsymbol{r}, t)-V_{\mathrm{C}}\right] \quad \text { for } V_{\mathrm{d}}>V_{\mathrm{C}}, \\
& =0 \quad \text { for } V_{\mathrm{d}}<V_{\mathrm{C}},
\end{aligned}
$$

where $V_{\mathrm{d}}(\boldsymbol{r}, t)=|\boldsymbol{J}(\boldsymbol{r}, t) / \rho(\boldsymbol{r}, t)|$ is the relative electron-ion drift velocity. Here, we take $k_{\mathrm{d}}=0.003$ and $V_{\mathrm{C}}=12$ in Eq. (4) as before. Also, in order to disturb the initial configuration, a localized resistivity is assumed around the origin $(\boldsymbol{r}=0)$ in the form,

$\eta(\boldsymbol{r})=\eta_{0} \exp \left[-\left(x / k_{\mathrm{x}}\right)^{2}-\left(y / k_{\mathrm{y}}\right)^{4}-\left(z / k_{\mathrm{z}}\right)^{4}\right]$,

which breaks the initial static condition and causes magnetic reconnection as an initial disturbance. Here, we take $\eta_{0}=0.02$ and $k_{\mathrm{x}}=k_{\mathrm{z}}=0.8$, and $k_{\mathrm{y}}=5$. This disturbance (5) is imposed only in the initial time range $0<t<4$, and the resistivity model (4) is assumed for $t>4$.
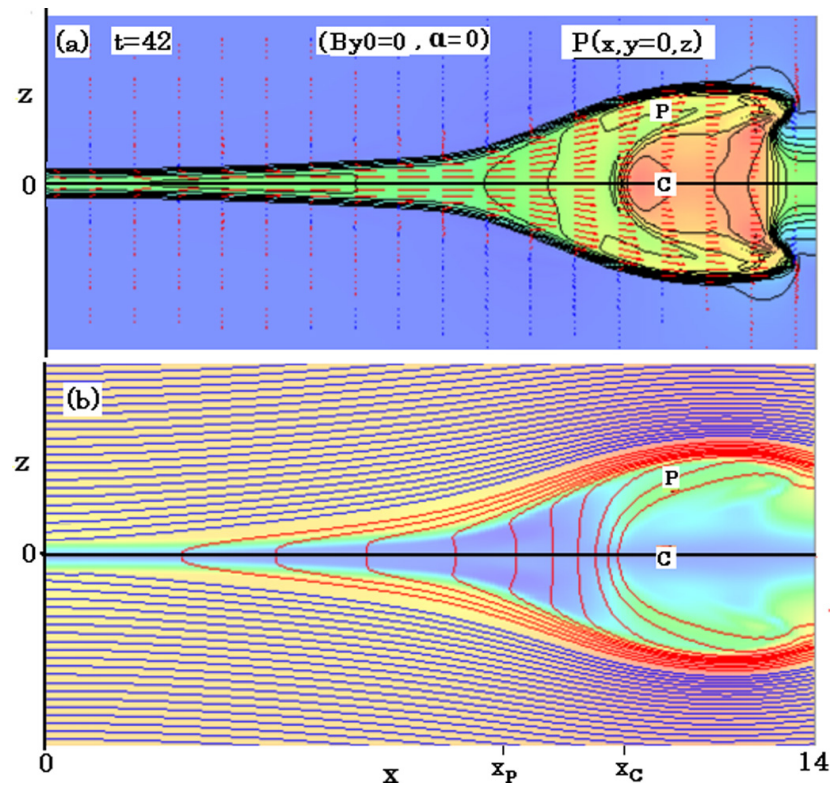

Fig. 2. (a) Plasma flow vectors and plasma pressure disribution with contour interval of 0.15 , and (b) magnetic field lines passing through the $\mathrm{x}$ - and z-axes with the distribution of $\left|B_{\mathrm{X}}\right|$ in color, in the $y=0[(x, z)]$ plane at time $t=42$ for the case of $B_{\mathrm{y} 0}=\alpha=$ 0 ; here, $X_{\mathrm{P}}$ and $X_{\mathrm{C}}$ indicate the plasmoid backward end and the plasmoid center, respectively, and $\mathrm{P}$ and $\mathrm{C}$ indicate, respectively, the plasmoid reconnection region and the plasmoid core region. The color from blue to red indicates the quantity magnitude from its minimum to the maximum; in what follows, all the similar figures are shown in this way.

The parameters for the numerical computations are taken to be $L_{\mathrm{x}}=14$ or $20, L_{\mathrm{z}}=7.2$, and $L_{\mathrm{y}}=10$. The mesh sizes are $\Delta x=0.04, \Delta z=0.015$ and $\Delta y=0.1$.

\section{Results}

Because of the present symmetry boundary conditions, the plasmoids propagate both in the positive and negative $\mathrm{x}$ directions. In what follows, only the plasmoid propagating in the positive $\mathrm{x}$-direction will be shown. First of all, the basic plasmoid structure in the absence of initial sheared field is shown, which is consistent with those reported in our previous 2-D and three-dimensional (3-D) MHD simulations (Ugai and Zheng, 2006, and references therein). Next, we demonstrate how the plasmoid structure is transformed by the initial sheared field in the current sheet to provide the definite magnetic field profiles inside the plasmoid.

\subsection{Plasmoid structure with no initial sheared field}

For the case of $\alpha=0$ and $B_{\mathrm{y} 0}=0$ (no initial sheared field), the overall configuration becomes symmetric with respect to the $y=0[(x, z)]$ and $z=0[(x, y)]$ planes. Figure 2 shows the 

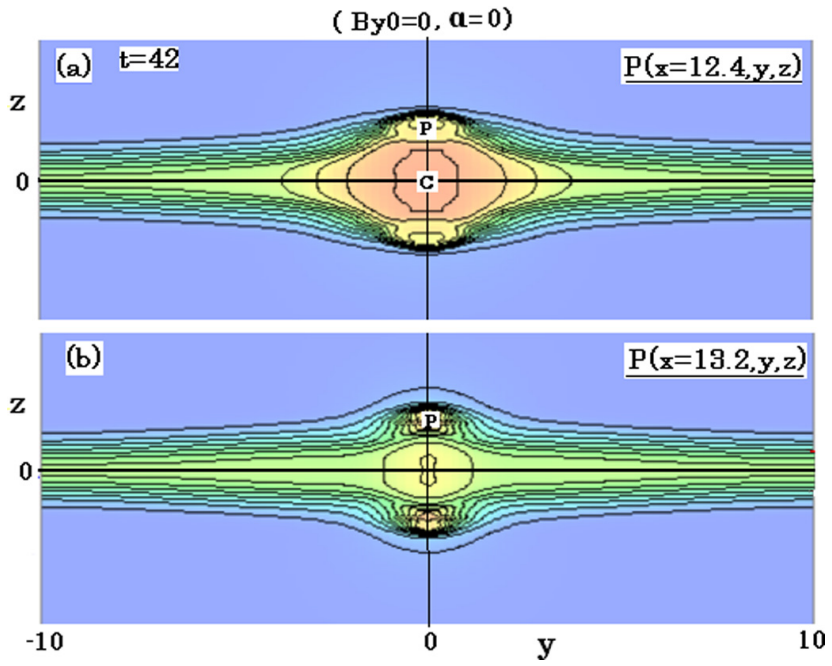

Fig. 3. Plasma pressure distributions with contour interval of 0.15 at $t=42$ for the case of $B_{\mathrm{y} 0}=\alpha=0$ in the cross sections at (a) $x=$ 12.4 and (b) $x=13.2$, where the regions $\mathrm{P}$ and $\mathrm{C}$ are indicated.

plasma flow velocity vectors with the plasma pressure distribution and the magnetic field configuration with the $B_{\mathrm{x}}$ field distribution in the $y=0[(x, z)]$ plane at time $t=42$, when the large-scale plasmoid has fully been established. Note that the magnetic field lines in the (x,z)-plane are coplanar. The plasmoid structure along the $\mathrm{x}$-axis is characterized by the backward end of the plasmoid, $X_{\mathrm{P}}$, and the plasmoid center, $X_{\mathrm{C}}$ (here, $L_{\mathrm{x}}=14$ is taken). In the extent $X_{\mathrm{P}}<x<X_{\mathrm{C}}$, the magnetized plasma with the reconnected field lines with $B_{\mathrm{Z}}>0$, which have been accelerated and heated by slow shocks, are accumulated, whereas in $X_{\mathrm{C}}<x$ the plasma, which was initially embedded in the current sheet and has been ejected away by the reconnection jet, is compressed and accumulated (Ugai, 2010a, b). Therefore, on the $\mathrm{x}$-axis, the reconnected field component $B_{\mathrm{Z}}>0$ in $X_{\mathrm{P}}<x<X_{\mathrm{C}}$, whereas $B_{\mathrm{Z}}=0$ in $X_{\mathrm{C}}<x$. Also, Fig. 3 shows the plasma pressure distributions in the cross sections at $x=12.4$ and $x=13.2$, which are taken to be larger than $X_{\mathrm{C}} \sim 11$. The plasma pressure in the $x=12.4\left(>X_{\mathrm{C}}\right)$ plane is distinctly enhanced in the middle region, denoted by $\mathrm{C}$, and in the spot-like regions above and below the region $\mathrm{C}$, denoted by $\mathrm{P}$. The plasmoid dimension is effectively confined in the $y$-direction, outside which there is little influence of reconnection, since the fast reconnection process is effectively limited in the y-direction (Ugai, 2009b).

In the absence of initial sheared field, the resulting plasmoid consists of high- $\beta$ plasma because of the strong plasma compression. On the basis of Figs. 2 and 3, the plsmoid structure may be schematically illustrated in Fig. 4, where the overall domain of the plasmoid, propagating in the positive $\mathrm{x}$-direction, is divided into the two characteristic regions $\mathrm{C}$ and $\mathrm{P}$ as follows. In the plasmoid core region $\mathrm{C}$, located in $x>X_{\mathrm{C}}$, the plasma, which was initially embed-

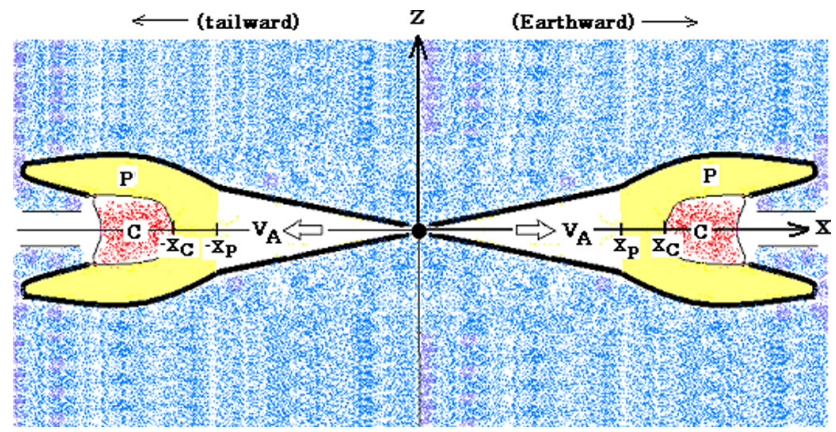

Fig. 4. Schematic drawing of a pair of plasmoids propagating in the positive and negative $\mathrm{x}$-directions in the $y=0[(x, z)]$ plane, where the overall plasmoid domain is divided into the plasmoid reconnection region $\mathrm{P}$ in yellow and the plasmoid core region $\mathrm{C}$ in red; here, $X_{\mathrm{P}}\left(-X_{\mathrm{P}}\right)$ and $X_{\mathrm{C}}\left(-X_{\mathrm{C}}\right)$ indicate, respectively, the plasmoid backward end and the plasmoid center, and $V_{\mathrm{A}}$ is the Alfvén velocity.

ded in the central current sheet and has been ejected away by the reconnection jet, is distinctly compressed and accumulated, so that the plasma density and pressure are enhanced. The plasmoid center $X_{\mathrm{C}}$ corresponds to the contact discontinuity that bounds the reconnected plasma and the plasma without reconnection. The region $\mathrm{C}$ is located in the center of the plasmoid and may hence be considered to be the plasmoid core. In the plasmoid reconnection region $\mathrm{P}$, the magnetized plasma with the reconnected field lines, which has been accelerated and heated by slow shocks, are accumulated. Hence, in this region the plasma flow velocities are considerably large, so that the region extends in the $\mathrm{x}$ direction like a nose beyond the region C (Fig. 2); also, as shown in Fig. 3b, in the cross section of the nose at $x=13.2$ (Fig. 2a), a pair of (upper and lower) spots of the region $\mathrm{P}$ are formed ahead of the region $\mathrm{C}$.

\subsection{Plasmoid structure in a sheared field}

In the absence of the initial sheared field, the plasmoid reconnection region $\mathrm{P}$ with the reconnected field lines is located exactly above and below the plasmoid core region $\mathrm{C}$ for $x>X_{\mathrm{C}}$ (Fig. 3). With this in mind, let us consider how the basic plasmoid structure is transformed by the sheared field. In general, we find that the plasmoid evolution is, at least qualitatively, not influenced by the magnitude of the initial sheared field (Ugai, 2010a, b). Hence, the results will be demonstrated mainly for the typical case of $B_{\mathrm{y} 0}=0.1$ and $\alpha=0.1\left(B_{\mathrm{y}}>0\right)$. Figure 5 shows for this case the magnetic field lines projected onto the $y=0[(x, z)]$ plane, the distribution of the magnetic field component $B_{\mathrm{y}}$ and that of the plasma pressure $P$ in the $(x, z)$ plane at time $t=45$, when the plasmoid is fully established (here, $L_{\mathrm{X}}=20$ is taken). Also, Fig. 6 shows the profiles of plasma density $\rho$ and field components $B_{\mathrm{y}}$ and $B_{\mathrm{z}}$ along the x-axis around the 

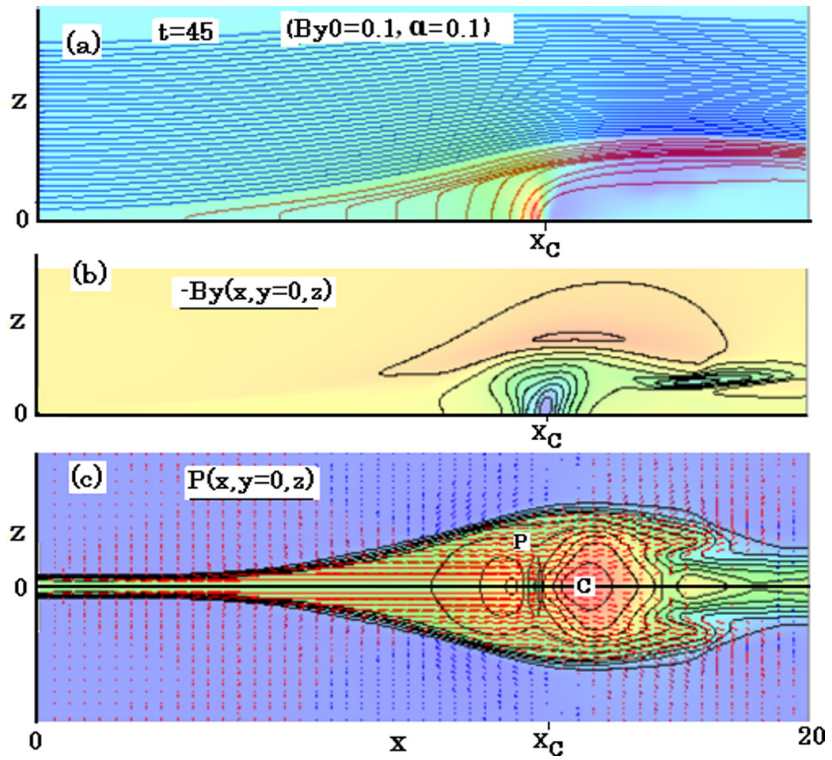

Fig. 5. (a) Magnetic field lines passing through the $\mathrm{x}$ - and $\mathrm{z}$-axes, projected onto the $y=0[(x, z)]$ plane, with the distribution of $B_{\mathrm{Z}}$ in color, (b) distribution of $-B_{\mathrm{y}}$ with contour interval of 0.1 and (c) plasma pressure distribution with contour interval of 0.15 and plasma flow vectors, in the $y=0$ plane at $t=45$ for the case of $B_{\mathrm{y} 0}=0.1$ and $\alpha=0.1$, where $X_{\mathrm{C}}$ is the plasmoid center and the regions $\mathrm{P}$ and $\mathrm{C}$ are indicated.

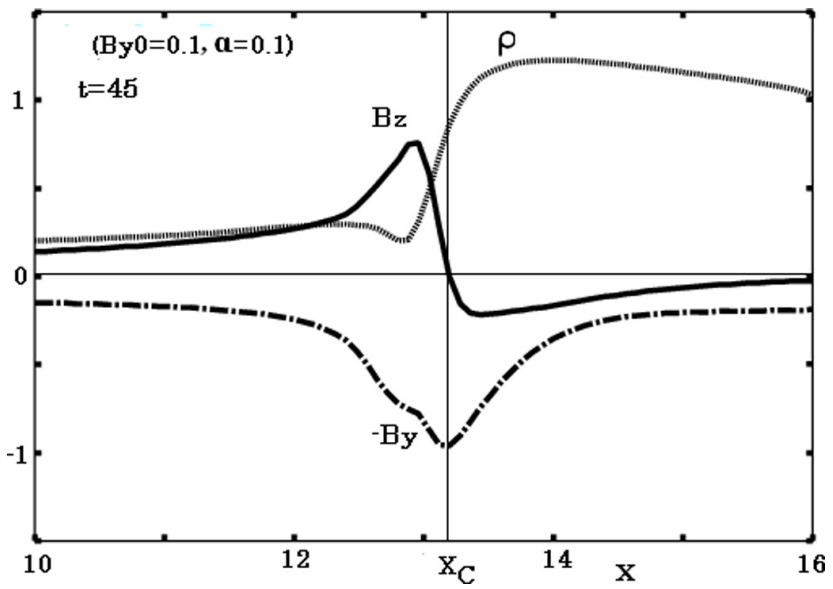

Fig. 6. Profiles of the plasma density $\rho,-B_{\mathrm{y}}$ and $B_{\mathrm{Z}}$ at $t=45$ along the X-axis around the plasmoid center $X_{\mathrm{C}}$ for the case of $B_{\mathrm{y} 0}=0.1$ and $\alpha=0.1$.

plasmoid center $X_{\mathrm{C}}$. As already shown, $\left|B_{\mathrm{y}}\right|$ has the maximum value at $x=X_{\mathrm{C}}$, where the $B_{\mathrm{z}}$ field changes its sign and the plasma density $\rho$ increases because of the plasma compression around $x=X_{\mathrm{C}}$. In this case too, the plasmoid center $X_{\mathrm{C}}$ corresponds to the contact discontinuity that bounds the reconnected field lines and those without reconnection (Ugai, 2010a, b).

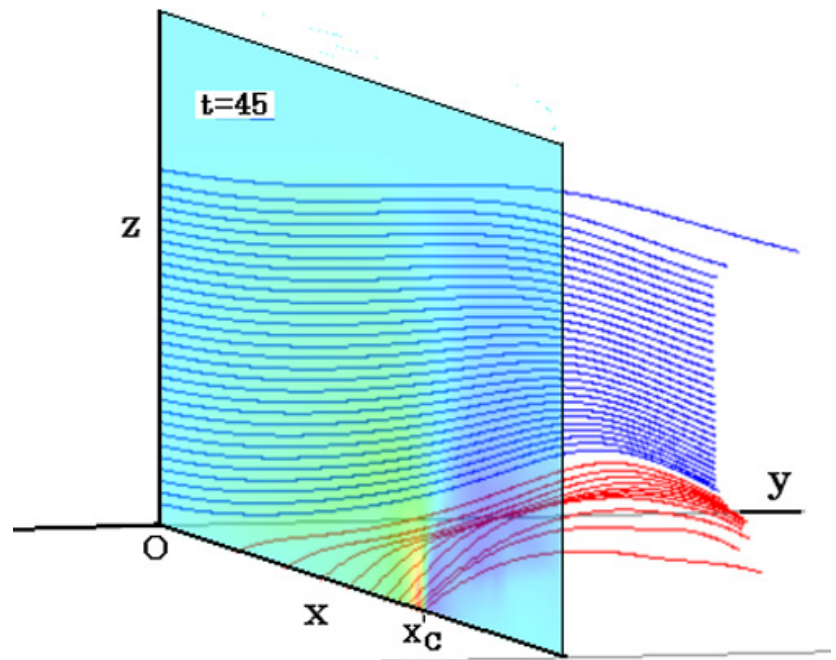

Fig. 7. Magnetic field lines passing through the z-axis (blue) and the reconnected field lines passing through the $\mathrm{x}$-axis (red), and distribution of $B_{\mathrm{Z}}$ in color in the $y=0[(x, z)]$ plane, at $t=45$ for the case of $B_{\mathrm{y} 0}=0.1$ and $\alpha=0.1$, where $X_{\mathrm{C}}$ indicates the plasmoid center.

As in Fig. 4, the resulting plasmoid is divided into the regions $\mathrm{P}$ and $\mathrm{C}$ as shown in Fig. 5c. In this case, the sheared field lines $\left(B_{\mathrm{y}}\right)$ are embedded in the initial current sheet, so that the magnetized plasma with the sheared field lines has been ejected away by the reconnection jet and are accumulated in the core region $\mathrm{C}$ in $x>X_{\mathrm{C}}$. Note that the sheared field lines may continuously be accumulated in the region $\mathrm{C}$ as the plasmoid (the region $\mathrm{C}$ ) propagates in the positive $\mathrm{X}$ direction, since the field lines are frozen-into the plasma. In the reconnection region $\mathrm{P}$, the magnetized plasma with the reconnected sheared field lines is accumulated, so that the $B_{\mathrm{y}}$ field is notably compressed around $x=X_{\mathrm{C}}$ (Figs. 5b and $6)$. Figure 7 shows the magnetic field lines passing through the $\mathrm{z}$-axis and the reconnected field lines passing through the $\mathrm{X}$-axis, which are the same as Fig. 5a but viewed from another angle. Apparently, the reconnected field lines passing through the $x$ axis in $x<X_{\mathrm{C}}$, which have the field component $B_{\mathrm{z}}>0$, are significantly skewed in the positive $\mathrm{y}$-direction in $z>0$.

The reconnected field lines are notably skewed (Fig. 7), so that the plasmoid structure may accordingly be distorted, since the plasma flows tend to be directed along the field lines. Figure 8 shows the plasma pressure distributions in the cross sections at $x=13.2,13.6$ and $x=17.6\left(X_{\mathrm{C}} \sim 13.2\right.$ in Fig. 6). We readily see that the region $\mathrm{P}$, where the plasma pressure is enhanced, is significantly shifted in the positive $y$-direction in $z>0$ and in the negative $y$-direction in $z<0$ because of the $\mathrm{x}$-axis symmetry. Also, Fig. 9 shows the distributions of the $B_{\mathrm{z}}$ and $B_{\mathrm{y}}$ field components in the $x=$ 13.2 $\left(\sim X_{\mathrm{C}}\right)$ plane. Around the plasmoid center $(y=z=0)$ $B_{\mathrm{z}} \sim 0$ and $\left|B_{\mathrm{y}}\right|$ has the maximum value in the form of a core 

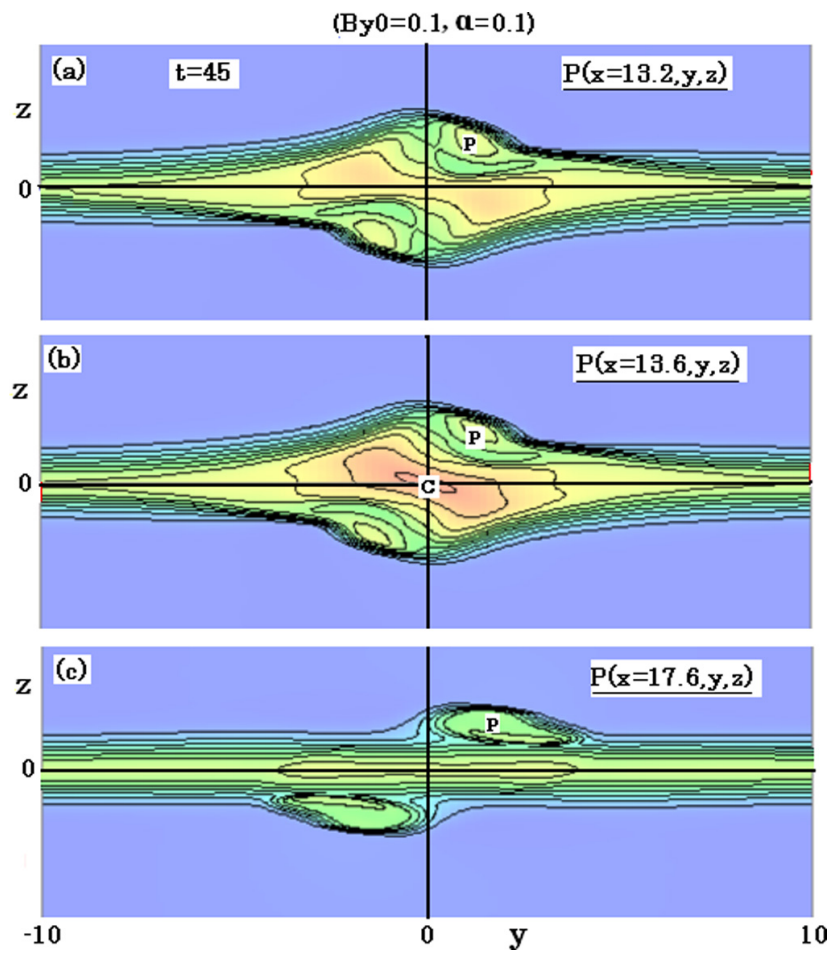

Fig. 8. Plasma pressure distributions (contour interval of 0.15) at $t=45$ for the case of $B_{\mathrm{y} 0}=0.1$ and $\alpha=0.1$ in the cross sections at (a) $x=13.2$, (b) $x=13.6$ and (c) $x=17.6$, where the regions $\mathrm{P}$ and $\mathrm{C}$ are indicated.

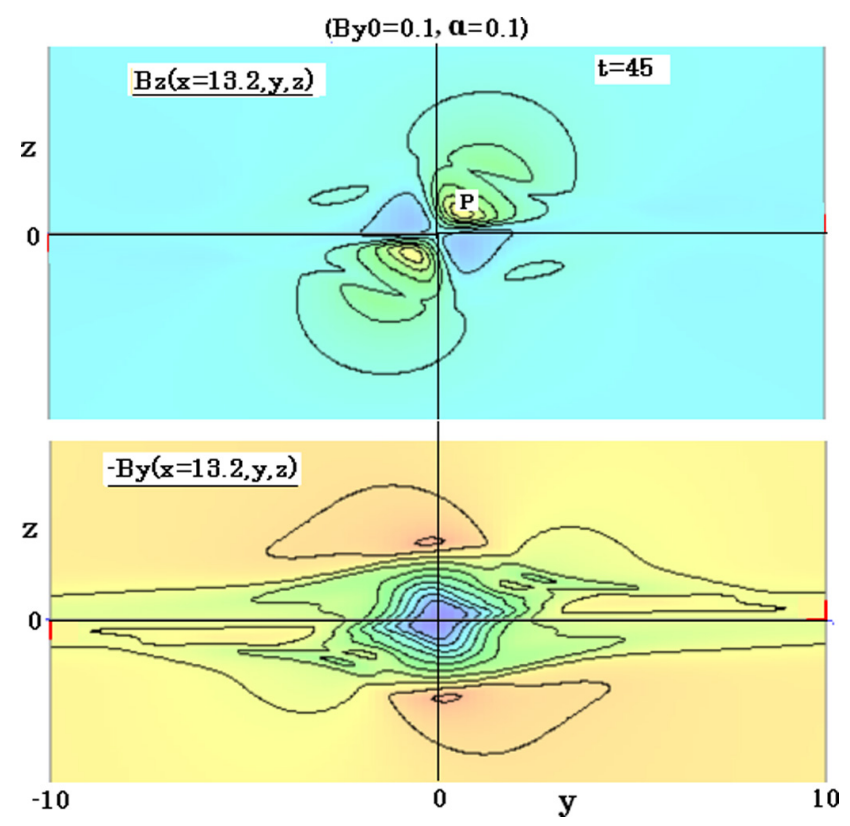

Fig. 9. Distributions of $B_{\mathrm{z}}$ and $-B_{\mathrm{y}}$ (contour interval of 0.1 ) at $t=45$ for the case of $B_{\mathrm{y} 0}=0.1$ and $\alpha=0.1$ in the cross section at $x=13.2$.
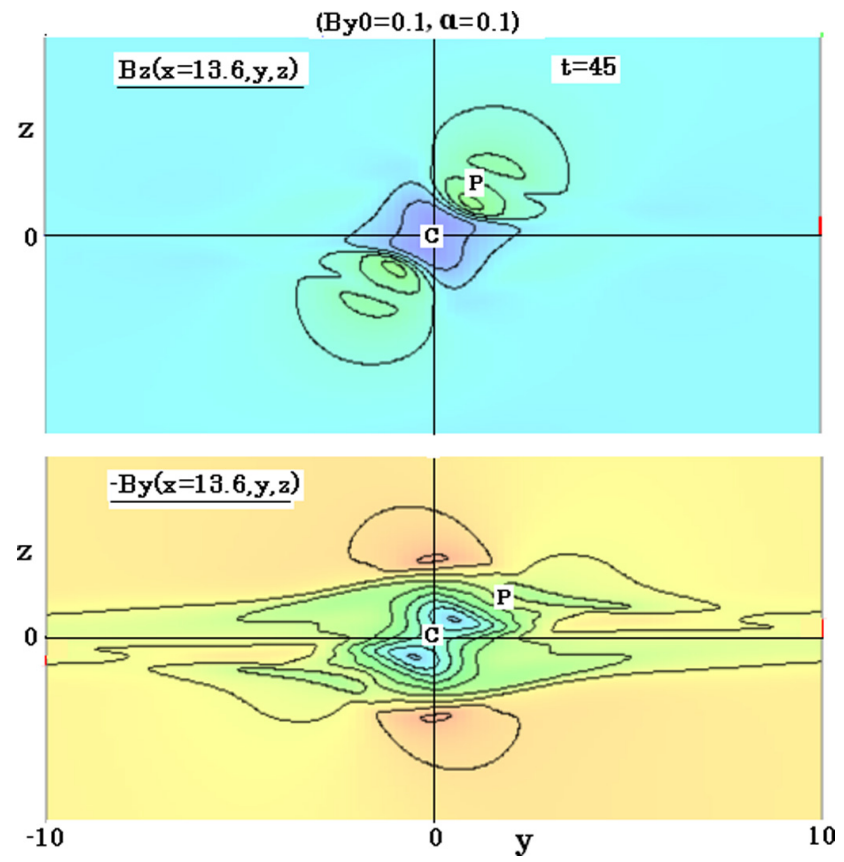

Fig. 10. Distributions of $B_{\mathrm{Z}}$ and $-B_{\mathrm{y}}$ (contour interval of 0.1 ) at $t=45$ for the case of $B_{\mathrm{y} 0}=0.1$ and $\alpha=0.1$ in the cross section at $x=13.6$, where the regions $\mathrm{P}$ and $\mathrm{C}$ are indicated.

(Fig. 6). Similarly, Fig. 10 shows the distributions of the $B_{\mathrm{z}}$ and $B_{\mathrm{y}}$ field components in the $x=13.6$ plane just ahead of the plasmoid center $X_{\mathrm{C}}$. This figure shows that in the plasmoid core region $C$ (around $y=z=0$ ), where the sheared field lines are accumulated, the $B_{\mathrm{Z}}$ field becomes definitely negative (Fig. 6). Also, the location of $x=17.6$ corresponds to the nose of the reconnection region $\mathrm{P}$ (Fig. 5), where the spot-like parts of the region $\mathrm{P}$, located above and below the $\mathrm{x}$-axis, are shifted in the positive and negative $y$-directions, respectively (Fig. 8c).

Let us interpret how the $B_{\mathrm{Z}}$ field becomes negative just ahead of $x=X_{\mathrm{C}}$ to have the bipolar profile around the plasmoid center $X_{\mathrm{C}}$ (Fig. 6). In the presence of the initial sheared field, the sheared field lines $\left(B_{\mathrm{y}}>0\right)$ are continuously accumulated in the plasmoid core region $C$; simultaneously, the reconnected field lines with the sheared field $B_{\mathrm{y}}>0$ are compressed along the $\mathrm{x}$-axis in $x<X_{\mathrm{C}}$ in the region $\mathrm{P}$, so that the field component $B_{\mathrm{y}}$ may have the peak value at the plasmoid center $X_{\mathrm{C}}$ at the interface between the regions $\mathrm{P}$ and $C$ (Fig. 4). In this situation, the reconnection region $P$ of enhanced plasma pressure is shifted in the positive $\mathrm{y}$ direction above the region $\mathrm{C}$ and in the negative $\mathrm{y}$-direction below the region $\mathrm{C}$ (Fig. 8b). Then, as seen in Fig. 8b, the (shifted) upper part of the region $\mathrm{P}$ above the region $\mathrm{C}$ tends to push the region $\mathrm{C}$ in the negative $\mathrm{z}$-direction, whereas the (shifted) lower part of the region $\mathrm{P}$ below the region $\mathrm{C}$ tends to push the region $\mathrm{C}$ in the positive z-direction. In this manner, the region $\mathrm{C}$ in the $x=13.6$ plane just ahead 

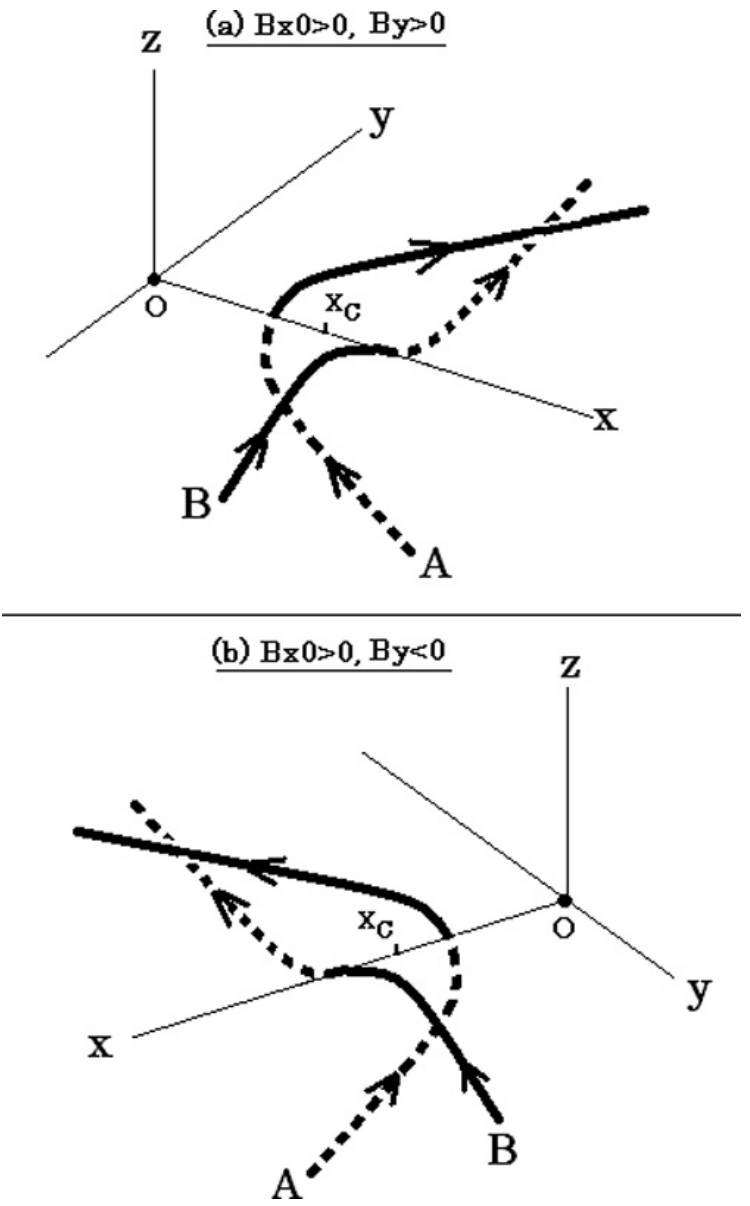

Fig. 11. Schematic drawings of the reconnected field lines, represented by $\boldsymbol{A}$, which pass through the x-axis in $x<X_{\mathrm{C}}$ and the plasmoid reconnection region $P$, and the accumulated sheared field lines, represented by $\boldsymbol{B}$, which pass through the x-axis in $x>X_{\mathrm{C}}$ and the plasmoid core region $C$, where dotted lines indicate the field lines in $z<0$, for the cases (a) $B_{\mathrm{x} 0}>0, B_{\mathrm{y}}>0$ and (b) $B_{\mathrm{x} 0}>0, B_{\mathrm{y}}<0$.

of the plasmoid center may be bent in the z-direction around $y=z=0$ (Fig. 8b). Therefore, the sheared magnetic field lines with $B_{\mathrm{y}}>0$, which are accumulated in the core region $\mathrm{C}$, should simultaneously be bent, leading to $B_{\mathrm{Z}}<0$ in the region $\mathrm{C}$ at $x=13.6$ (Figs. 6 and 10).

In order to see more clearly the complicated 3-D magnetic field topology in the plasmoid, Fig. 11 schematically illustrates the typical field lines passing inside the plasmoid. For the case of $B_{\mathrm{x} 0}>0$ and $B_{\mathrm{y}}>0$, as shown in Fig. 11a, the reconnected field lines, denoted by $\boldsymbol{A}$, come from the negative $\mathrm{z}$ - and negative $\mathrm{y}$-directions, pass through the $\mathrm{x}$-axis in $x<X_{\mathrm{C}}$ and go to the positive $\mathrm{z}$ - and positive $\mathrm{y}$-directions. The reconnected field lines $\boldsymbol{A}$ pass through the plasmoid reconnection region $\mathrm{P}$, which is now shifted in the positive $\mathrm{y}$ direction in $z>0$ and in the negative $y$ direction in $z<0$ in $x>X_{\mathrm{C}}$ (Fig. 8). On the other hand, the accumulated sheared field lines, denoted by $\boldsymbol{B}$, are basically directed to the posi-
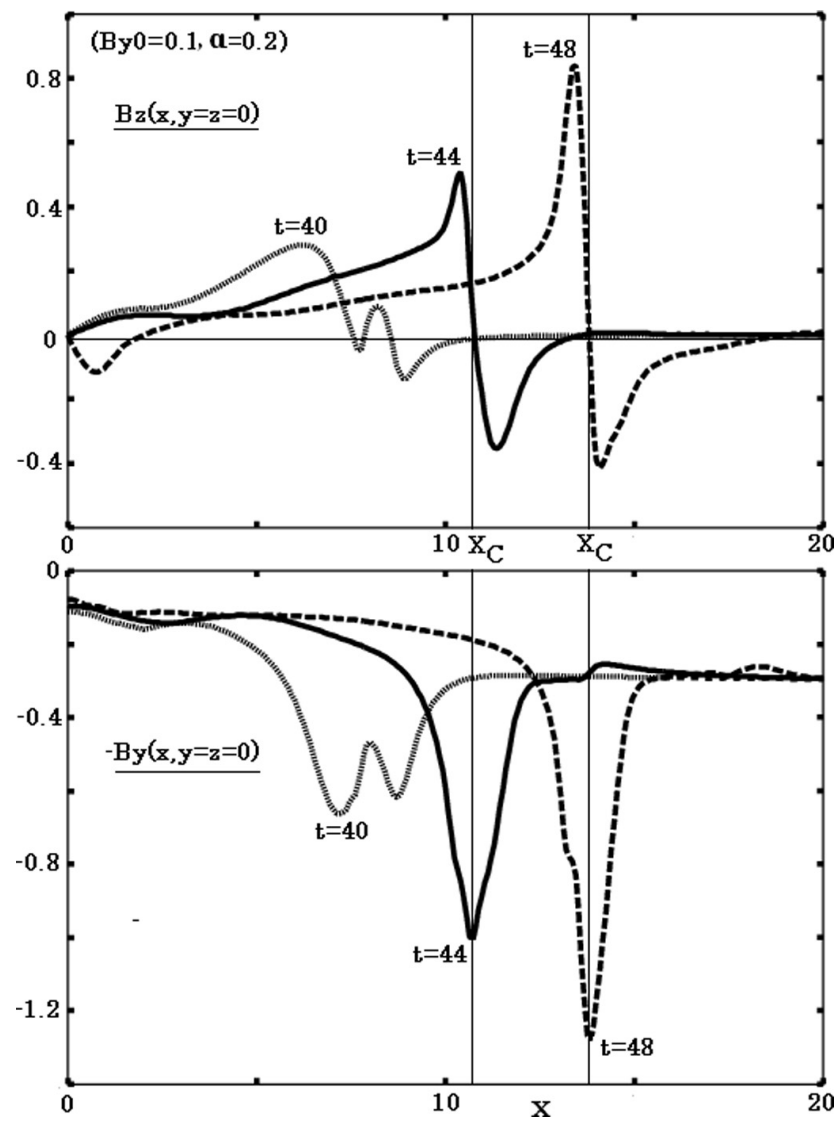

Fig. 12. Profiles of $B_{\mathrm{Z}}$ and $-B_{\mathrm{y}}$ along the $\mathrm{x}$-axis at different times for the case of $B_{\mathrm{y} 0}=0.1$ and $\alpha=0.2$.

tive y-direction and pass through the plasmoid core region $\mathrm{C}$ in $x>X_{\mathrm{C}}$. Since the region $\mathrm{P}$ of enhanced pressure, shifted in the $y$-directions, tends to push the core region $\mathrm{C}$ in $x>X_{\mathrm{C}}$ downward in $y>0$ and upward in $y<0$, the sheared filed lines $\boldsymbol{B}$ are forced to be bent in the z-direction to cause the field component $B_{\mathrm{Z}}<0$ around the x-axis (see also Fig. 10). For the case of $B_{\mathrm{y}}<0$ and $B_{\mathrm{x} 0}>0$, as shown in Fig. 11b, the sheared field lines $\boldsymbol{B}$, accumulated in the core region $\mathrm{C}$ in $x>X_{\mathrm{C}}$, are forced to be bent in the z-direction because of the $\mathrm{y}$-directional shift of the region $\mathrm{P}$, giving rise to $B_{\mathrm{Z}}<0$ around the $\mathrm{x}$-axis (in this case, the region $\mathrm{P}$ is shifted in the positive $\mathrm{y}$-direction in $z<0$ and negative $\mathrm{y}$-direction in $z>0$ ). In any case, the sign of the $B_{Z}$ component of the reconnected field lines $\boldsymbol{A}$ in $x<X_{\mathrm{C}}$ is opposite to that of the field lines of $\boldsymbol{B}$ in $x>X_{\mathrm{C}}$. Hence, independently of the sign of the sheared field $\left(B_{\mathrm{y}}\right)$ initially embedded in the current sheet, the $B_{\mathrm{Z}}$ field component always changes its sign across the resulting plasmoid center $X_{C}$, giving rise to the definite bipolar profile.

Finally, let us briefly exemplify some typical results for different parameters. Figure 12 shows for the case of $B_{\mathrm{y} 0}=$ 0.1 and $\alpha=0.2$ the profiles of $B_{\mathrm{z}}$ and $B_{\mathrm{y}}$ along the x-axis at different times. In this case, the initial sheared field is 
$(\mathrm{By} 0=0.1, \mathbf{a}=0.2)$
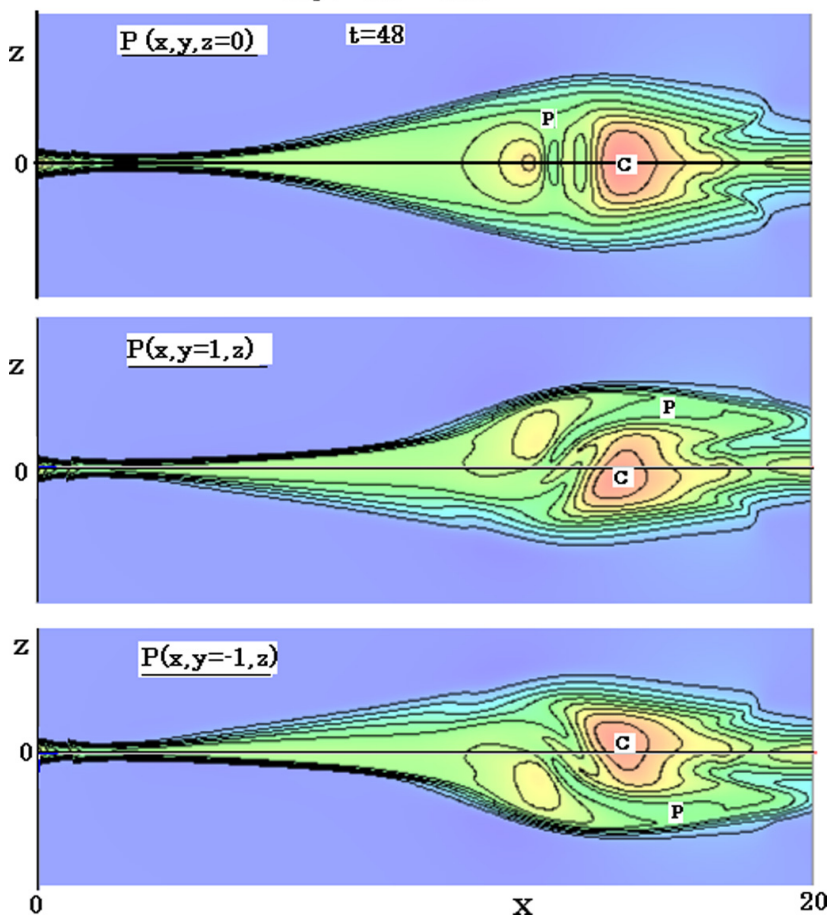

Fig. 13. Plasma pressure distributions (contour interval of 0.15 ) at $t=48$ in the $y=0, y=1$ and $y=-1$ planes for the case of $B_{\mathrm{y} 0}=0.1$ and $\alpha=0.2$, where the regions $\mathrm{P}$ and $\mathrm{C}$ are indicated.

somewhat larger than in the previous case. At time $t=40$, when the plasmoid is not yet established, there are two humps of the $B_{\mathrm{y}}$ field: One is associated with the reconnected field lines and the other at the larger $x$ location results from the compression of the sheared field lines initially embedded in the current sheet. At $t=44$ and $t=48$, when the plasmoid is fully established, the two humps have been combined at the plasmoid center $x=X_{\mathrm{C}}$, where $B_{\mathrm{y}}$ has the maximum value and $B_{\mathrm{Z}}$ changes its sign. Figure 13 shows the plasma pressure distributions at $t=48$ in the $y=0, y=1$ and $y=-1$ planes, where the plasmoid reconnection region $\mathrm{P}$ is distorted in the y-direction (Fig. 11a). Also, Fig. 14 shows the distributions of plasma pressure $P$ and magnetic field component $B_{\mathrm{Z}}$ in the $x=14.4$ plane, located just ahead of the plasmoid center $X_{\mathrm{C}} \sim 13.8$ (Fig. 12). As indicated in Fig. 11a, the plasmoid core region $\mathrm{C}$ is bent in the $\mathrm{z}$-direction, giving rise to $B_{\mathrm{Z}}<0$ in C (Fig. 14b). For the other parameter values, such as $B_{\mathrm{y} 0}=0.2$ and $\alpha=0$, the results are found to be, at least qualitatively, quite the same. Quantitatively, we generally find that as the accumulated sheared fields in the core region $\mathrm{C}$ become larger, the bipolar change in $B_{\mathrm{Z}}$ becomes more distinct (Fig. 12). This can readily be understood, since the bipolar $B_{\mathrm{Z}}$ profile results directly from the bending of the field lines $\boldsymbol{B}$ accumulated in $\mathrm{C}$ for $x>X_{\mathrm{C}}$ (Fig. 11).
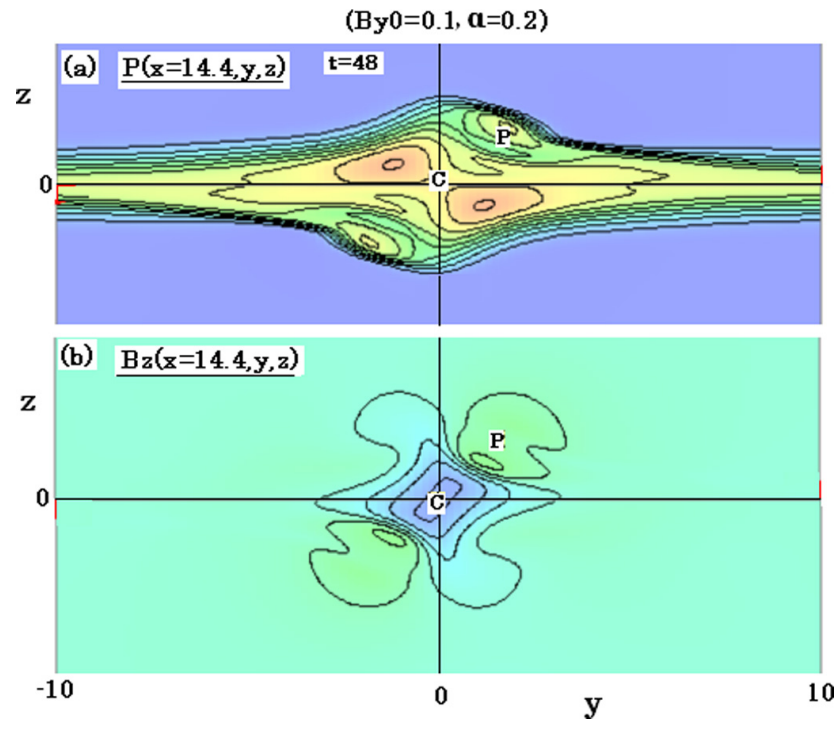

Fig. 14. Distributions of the plasma pressure $P$ (contour interval of 0.15 ) and $B_{\mathrm{Z}}$ (contour interval of 0.1 ) in the cross section at $x=14.4$ at $t=48$ for the case of $B_{\mathrm{y} 0}=0.1$ and $\alpha=0.2$, where the regions $\mathrm{P}$ and $\mathrm{C}$ are indicated.

\section{Summary and discussion}

Recently, the basic magnetic field profiles of large-scale plasmoid, generated by the fast reconnection mechanism, is studied (Ugai, 2010b), and it is demonstrated that they are, both qualitatively and quantitatively, in good agreement with the satellite observations (Ieda et al., 1998; Slavin et al., 2003) and, in particular, with those of the so-called night-side flux transfer events (Sergeev et al., 1992; Sormakov and Sergeev, 2008; Runov et al., 2009); outside the plasmoid too, the magnetic field profiles are consistent with the TCR signatures (Slavin et al., 1984, 1993; Ugai and Zheng, 2006). As a sequence of Ugai (2010b), the present paper studies the structure and dynamics of large-scale plasmoid in detail. As illustrated in Fig. 4, the overall plasmoid domain is divided into the plasmoid reconnection region $\mathrm{P}$ and the plasmoid core region $\mathrm{C}$. In the region $\mathrm{P}$, the magnetized plasma with reconnected field lines are compressed and accumulated, whereas in the region $\mathrm{C}$, the plasma, which was intially embedded in the current sheet and has been ejected away by the reconnection jet, is compressed and accumulated. In the absence of the initial sheared field, the region $\mathrm{P}$ is located exactly above and below the region $\mathrm{C}$ for $x>X_{\mathrm{C}}$ (Fig. 3), and inside the region $\mathrm{C} B_{\mathrm{Z}}=0$ on the $\mathrm{x}$-axis. In the presence of initial sheared field, the reconnected field lines are sheared in the reconnection region $\mathrm{P}$; also, in the core region $\mathrm{C}$, the sheared field lines, initially embedded in the current sheet, are accumulated, since the field lines are frozen-into the current sheet plasma. The plasmoid center $X_{\mathrm{C}}$ on the x-axis, located at the interface between the regions $\mathrm{P}$ and $\mathrm{C}$, corresponds to the 
contact discontinuity that bounds the reconnected field lines in $\mathrm{P}$ and the field lines without reconnection in $\mathrm{C}$.

For the case of the initial sheared field $B_{\mathrm{y}}>0$, the upper (lower) part of the plasmoid reconnection region $\mathrm{P}$ propagating in the positive $\mathrm{x}$-direction is shifted in the positive (negative) y-direction (Fig. 8). The field lines passing through the regions $\mathrm{P}$ and $\mathrm{C}$ are illustrated in Fig. 11. As seen from this figure, the region $\mathrm{P}$ shifted in the $\mathrm{y}$-direction causes the plasmoid core region $\mathrm{C}$ with the accumulated sheared field lines to be bent in the $\mathrm{z}$-direction around the $\mathrm{x}$-axis in $x>X_{\mathrm{C}}$ (Figs. 8b and 14a), leading to the field component $B_{\mathrm{Z}}<0$ just ahead of $x=X_{\mathrm{C}}$ (Figs. 6 and 12). For the case of $B_{\mathrm{y}}<0$ too, the bending of the region $C$ causes $B_{\mathrm{Z}}<0$ ahead of $X_{\mathrm{C}}$. Therefore, if $B_{\mathrm{Z}}=0$ initially, independently of the sign of the initial sheared field $B_{\mathrm{y}}$, the field component $B_{\mathrm{z}}$ always changes its sign across the plasmoid center $X_{\mathrm{C}}$, leading to the definite bipolar profile. The bipolar profile of $B_{\mathrm{Z}}$ may become more distinct as the sheared magnetic fields are more accumulated in the region $C$ (Fig. 12), since the field component $B_{\mathrm{Z}}<0$ results directly from the accumulated sheared magnetic field lines in the region $C$.

Unlike the traditional plasmoid picture, we have demonstrated that even if there is no northward magnetic field in the initial current sheet, the bipolar profile of $B_{\mathrm{Z}}$ is caused across the plasmoid center $X_{\mathrm{C}}$. This implies that the initial northward magnetic field in the tail current sheet plays no essential role in the basic plasmoid structure or dynamics. This may be understood if one considers the effect of the initial northward field on the plasmoid dynamics as follows. Since the magnetized plasma initially in the reconnection outflow region is accumulated in the plasmoid core region $\mathrm{C}$, the only significant effect of the initial northward field component may arise in the region $\mathrm{C}$, where the accumulated sheared field lines may retain the initial northward field component. As shown in Fig. 4, plasmoids propagate in the Earthward $(x>0)$ and tailward $(x<0)$ directions. In the Earthward-moving plasmoid, the reconnected field lines in $x<X_{\mathrm{C}}$ have the northward field component $\left(B_{\mathrm{Z}}>0\right)$, whereas the accumulated field lines in $C$ are bent so as to enhance the southward field component $\left(B_{\mathrm{Z}}<0\right)$ in $x>X_{\mathrm{C}}$ (Fig. 6 or 12). Hence, the northward field component preexisting in $\mathrm{C}$ should be reduced in $x>X_{\mathrm{C}}$, and if the initial $B_{\mathrm{Z}}>0$ is sufficiently small, $B_{\mathrm{Z}}$ may become negative (southward) in $x>X_{\mathrm{C}}$. In the tailward-moving plasmoid, the reconnected field lines in $x>-X_{\mathrm{C}}$ have the southward field component $\left(B_{\mathrm{Z}}<0\right)$, whereas the bending of the accumulated field lines in $\mathrm{C}$ causes the northward field component $\left(B_{\mathrm{Z}}>0\right)$ in $x<-X_{\mathrm{C}}$ because of the symmety boundary condition, $B_{\mathrm{Z}}(-x, y=z=0)=-B_{\mathrm{Z}}(x, y=z=0)$. Hence, the northward field component pre-existing in $\mathrm{C}$ may enhance the northward component in $x<-X_{\mathrm{C}}$, leading to the bipolar profile of $B_{\mathrm{Z}}$ more symmetric. Also, it may be noted that even if the initial sheared field is small, the sheared field lines are continuously piled up inside the plasmoid core region $\mathrm{C}$ as the plasmoid propagates, so that the sheared field $\left(B_{\mathrm{y}}\right)$ can become very large (even larger than the lobe field) at the plasmoid center $X_{\mathrm{C}}$ (Fig. 12). These basic features seem to be generally consistent with satellite observations.

In summary, on the basis of the spontaneous fast reconnection model, we may suggest the following scenario for the plasmoid dynamics in the geomagnetic tail. The basic configuration of the tail current sheet should be controlled by solar wind conditions. Then, as shown in Fig. 1, once a sufficiently thin current sheet, say $W>3 d_{0}$, is formed somewhere in the tail, the fast reconnection mechanism can suddenly build up there. If the fast reconnection is fully established, large-scale plasmoids are generated and propagate in the tailward and Earthward directions (Fig. 4). The tailward-moving plasmoid can freely propagate down the geomagentic tail. In addition to the large-scale (principal) plasmoid, small-scale magnetic islands are likely to be generated in the active $\mathrm{X}$ reconnection (diffusion) region, which may also propagate in the tailward or Earthward direction (Ugai and Zheng, 2006). On the other hand, the Earthward-moving plasmoid eventually collides with the Earth's dipole field, which may cause many distinct phenomena. For instance, the so-called current wedge (McPherron et al., 1973) may be generated just ahead of the dipole field (say $|x| \sim 10 R_{\mathrm{E}}$ ) (Ugai, 2009a); also, in accordance with substorm onsets (Kamide, 1982), distinct magnetic pulsations of low frequency may occur (Ugai, 2009c). However, it should be noted that the fast reconnection evolution has very different aspects according to different situations; for instance, in critical situations, the fast reconnection mechanism cannot be fully established, which might lead to the so-called pseudosubstorms (Ugai, 2009b). Hence, in conclusion, it may be essential for the complicated substorm phenomena to understand the underlying physics of the fast reconnection mechanism.

Acknowledgements. The author thanks A. Nishida for useful discussions. This work was supported by the Grant-in-Aids (21340142) from the Ministry of Education in Japan, Mitsubishi Foundation, RISH of Kyoto University, and Solar-Terrestrial Environment Laboratory of Nagoya University.

Topical Editor I. A. Daglis thanks two anonymous referees for their help in evaluating this paper.

\section{References}

Abe, S. A. and Hoshino, M.: Nonlinear evolution of plasmoid structure, Earth, Planets Space, 53, 663-671, 2001.

Birn, J. and Hones Jr., E. W.: Three-dimensional computer modeling of dynamic reconnection in the geomagnetic tail, J. Geophys. Res., 86, 6802-6808, 1981.

Hones Jr., E. W.: Substorm processes in the magnetotail: Comments on "On hot tenuous plasma, fireballs, and boundary layers in the Earth's magnetotail” by L. A. Frank et al., J. Geophys. Res., 82, 5633-5640, 1977.

Ieda, A., Machida, S., Mukai, T., Saito, Y., Yamamoto, T., Nishida, A., Terasawa, T., and Kokubun, S.: Statistical analysis of the 
plasmoid evolution with Geotail observations, J. Geophys. Res., 103, 4453-4465, 1998.

Kamide, Y.: The relationship between field-aligned currents and the auroral electrojets - A review, Space Sci. Rev., 31, 127-243, 1982.

Klimchuk, J. A.: On solving the coronal heating problem, Solar Phys., 234, 41-77, 2006.

Lui, A. T. Y.: A multiscale model for substorms, Space Sci. Rev., 95, 325-345, 2001.

Lui, A. T. Y.: Potential plasma instabilities for substorm expansion onsets, Space Sci. Rev., 113, 127-206, 2004.

McPherron, R. L., Russel, C. T., and Aurbry, M. P.: Satellite studies of magnetospheric substorms on August 15, 1968. 9. Phenomenological model for substorms, J. Geophys. Res., 78, 31313149, 1973.

Nakamura, R., Baumjohann, W., Asano, Y., Runov, A., Balogh, A., Owen, C. J., Fazakerley, A. N., Fujimoto, M., Klecker, B., and Reme, H.: Dynamics of thin current sheets associated with magnetotail reconnection, J. Geophys. Res., 111, A11206, doi:10.1029/2006JA011706, 2006.

Nishida, A.: Can random reconnection on the magnetopause produce the low latitude boundary layer?, Geophys. Res. Lett., 16, 227-230, 1989.

Petkaki, P. and Freeman, M. P.: Nonlinear dependence of anomalous ion-acoustic resistivity on electron drift velocity, Astrophys. J., 686, 686-693, 2008.

Priest, E. R. and Forbes, T. G.: Magnetic Reconnection: MHD Theory and Applications, Cambridge University Press, Cambridge, UK, 2000.

Runov, A., Nakamura, R., Baumjohann, W., Treumann, R. A., Zhang, T. L., Volwerk, M., Voros, Z., Balogh, A., Glasmeier, K.H., Klecker, B., Reme, H., and Kistler, L.: Current sheet structure near magnetic X-line observed by Cluster, Geophys. Res. Lett., 30, 1579, doi:10.1029/2002GL016730, 2003.

Runov, A., Voronkov, I., Asano, Y., Baumjohann, W., Fujimoto, M., Nakamura, R., Takada, T., Volwerk, M., Vörös, Z., Meurant, M., Fazakerley, A., Rème, H., and Balogh, A.: Structure of the near-Earth plasma sheet during tailward flows, Ann. Geophys., 26, 709-724, doi:10.5194/angeo-26-709-2008, 2008.

Runov, A., Angelopoulos, V., Sitnov, M. I., Sergeev, V. A., Bonnell, J., McFadden, J. P., Larson, D., Glassmeier, K.H., and Auster, U.: THEMIS observations of an earthwardpropagating dipolarization front, Geophys. Res. Lett., 36, L14106, doi:10.1029/2009GL038980, 2009.

Sergeev, V. A., Elphic, R. C., Mozer, F. S., Saint-Marc, A., and Sauvaud, J. A.: A two-satellite study of nightside flux transfer events in the plasma sheet, Planet. Space Sci., 40, 1551-1572, 1992.

Sharma, A. S., Nakamura, R., Runov, A., Grigorenko, E. E., Hasegawa, H., Hoshino, M., Louarn, P., Owen, C. J., Petrukovich, A., Sauvaud, J.-A., Semenov, V. S., Sergeev, V. A., Slavin, J. A., Sonnerup, B. U. Ö., Zelenyi, L. M., Fruit, G., Haaland, S., Malova, H., and Snekvik, K.: Transient and localized processes in the magnetotail: a review, Ann. Geophys., 26, 9551006, doi:10.5194/angeo-26-955-2008, 2008.
Shirataka, N., Fujimoto, M., Hasegawa, H., and TanDokoro, R.: Reproducing the bipolar magnetic signature at the jet leading edge by three-dimensional reconnection with nonzero guide field, J. Geophys. Res., 111, A7201, doi:10.1029/2005JA011521, 2006.

Slavin, J. A., Smith, E. J., Tsurutani, B. T., Sibeck, D. G., Singer, H. J., Baker, D. N., Gosling, J. T., Hones, E. W., and Scarf, F. L.: Substorm associated traveling compression regions in the distant tail - ISEE-3 geotail observations, Geophys. Res. Lett., 11, 657660, 1984.

Slavin, J. A., Smith, M. F., Mazur, E. L., Baker, D. N., Hones Jr., E. W., Iyemori, T., and Greenstadt, E. W.: ISEE 3 observations of traveling compression regions in the Earth's magnetotail, J. Geophys. Res., 98, 15425-15446, 1993.

Slavin, J. A., Lepping, R. P., Gjerloev, J., Fairfield, D. H., Hesse, M., Owen, C. J., Moldwin, M. B., Nagai, T., Ieda, A., and Mukai, T.: Geotail observations of magnetic flux ropes in the plasma sheet, J. Geophys. Res., 108, 1015, doi:10.1029/93JA01467, 2003.

Sormakov, D. A. and Sergeev, V.: Topology of magnetic flux ropes in the magnetospheric plasma sheet as measured by the Geotail satellite, Cosmic Res., 46, 387-391, 2008.

Ugai, M.: Computer studies on plasmoid dynamics associated with the spontaneous fast reconnection mechanism, Phys. Plasmas, 2, 3320-3328, 1995.

Ugai, M.: The evolution of fast reconnection in a threedimensional current sheet system, Phys. Plasmas, 15, 082306, doi:10.1063/1.2969737, 2008.

Ugai, M.: Evolution of the current wedge and the generator current circuit by fast reconnection, Phys. Plasmas, 16, 012901, doi:10.1063/1.3055598, 2009a.

Ugai, M.: Fast reconnection evolution in an arcade-like magnetic loop structure, Phys. Plasmas, 16, 062312, doi:10.1063/1.3158949, 2009b.

Ugai, M.: Impulsive magnetic pulsations and electrojets in the loop footpoint driven by the fast reconnection mechanism, Phys. Plasmas, 16, 112902, doi:10.1063/1.3267869, 2009c.

Ugai, M.: Three-dimensional evolution of the fast reconnection mechanism in a force-free current sheet, Phys. Plasmas, 17, 06291, doi:10.1063/1.3431097, 2010a.

Ugai, M.: Magnetic field structure of large-scale plasmoid generated by the fast reconnection mechanism in a sheared current sheet, Ann. Geophys., 28, 1511-1521, doi:10.5194/angeo-281511-2010, 2010b.

Ugai, M. and Zheng, L.: Parametric studies on traveling compression regions observed in the Earth's magnetotail, Phys. Plasmas, 13, 062906, doi:10.1063/1.2212827, 2006. 